\title{
Magnetic fields and star formation in low-mass Magellanic-type and peculiar galaxies ${ }^{\star}$
}

\author{
W. Jurusik ${ }^{1}$, R. T. Drzazga ${ }^{1}$, M. Jableka ${ }^{1}$, K. T. Chyży ${ }^{1}$, R. Beck ${ }^{2}$, U. Klein ${ }^{3}$, and M. Weżgowiec ${ }^{4}$ \\ ${ }^{1}$ Obserwatorium Astronomiczne Uniwersytetu Jagiellońskiego, ul. Orla 171, 30-244 Kraków, Poland \\ e-mail: jurusik@oa.uj.edu.pl \\ 2 Max-Planck-Institut für Radioastronomie, Auf dem Hügel 69, 53121 Bonn, Germany \\ 3 Argelander-Institüt für Astronomie, Auf dem Hügel 71, 53121 Bonn, Germany \\ ${ }^{4}$ Astronomisches Institut der Ruhr-Universität Bochum, Universitätsstrasse 150, 44780 Bochum, Germany
}

Received 15 November 2013 / Accepted 6 June 2014

\section{ABSTRACT}

\begin{abstract}
Aims. We investigate how magnetic properties of Magellanic-type and perturbed objects are related to star-forming activity, galactic type, and mass.

Methods. We present radio and magnetic properties of five Magellanic-type and two peculiar low-mass galaxies observed at 4.85 and/or $8.35 \mathrm{GHz}$ with the Effelsberg $100 \mathrm{~m}$ telescope. The sample is extended to 17 objects by including five Magellanic-type galaxies and five dwarf ones.

Results. The distribution of the observed radio emission of low-mass galaxies at $4.85 / 8.35 \mathrm{GHz}$ is closely connected with the galactic optical discs, which are independent for unperturbed galaxies and those which show signs of tidal interactions. The strengths of total magnetic field are within $5-9 \mu \mathrm{G}$, while the ordered fields reach $1-2 \mu \mathrm{G}$, and both these values are larger than in typical dwarf galaxies and lower than in spirals. The magnetic field strengths in the extended sample of 17 low-mass galaxies are well correlated with the surface density of star formation rate (correlation coefficient of 0.87 ) and manifest a power-law relation with an exponent of $0.25 \pm 0.02$ extending a similar relation found for dwarf galaxies. We claim that the production of magnetic energy per supernova event is very similar for all the various galaxies. It constitutes about $3 \%\left(10^{49} \mathrm{erg}\right)$ of the individual supernovae energy release. We show that the total magnetic field energy in galaxies is almost linearly related to the galactic gas mass, which indicates equipartition of the magnetic energy and the turbulent kinetic energy of the interstellar medium. The Magellanic-type galaxies fit very well with the radio-infrared relation constructed for surface brightness of galaxies of various types, including bright spirals and interacting objects (with a slope of $0.96 \pm 0.03$ and correlation coefficient of 0.95 ). We found that the typical far-infrared relation based on luminosity of galaxies is tighter and steeper but more likely to inherit a partial correlation from a tendency that larger objects are also more luminous.

Conclusions. The estimated values of thermal fractions, radio spectral indices, and magnetic field strengths of the Magellanic-type galaxies are between the values determined for grand-design spirals and dwarf galaxies. The confirmed magnetic field-star formation and radio-infrared relations for low-mass galaxies point to similar physical processes that must be at work in all galaxies. More massive, larger galaxies have usually stronger magnetic fields and larger global star formation rates, but we show that their values of magnetic energy release per supernova explosion are still similar to those of dwarf galaxies.
\end{abstract}

Key words. galaxies: general - galaxies: magnetic fields - galaxies: interactions - radio continuum: galaxies

\section{Introduction}

While galactic magnetism has been known for over 30 years and observed for various galaxy types (e.g. Beck \& Wielebinski 2013), it is still far from being fully understood. Of particular importance are investigations concerning the generation and evolution of magnetic fields in low-mass objects, in which spiral density waves might be too weak to trigger star formation and influence magnetic fields. In such galaxies, due to their lower gravitational potential, other processes, such as galactic winds and stochastically propagating star formation, play a more important role. For these objects, we do not know the exact conditions required for the efficient amplification of the large-scale magnetic fields and the relations of magnetic fields to other phases of the interstellar medium (ISM) yet.

* Based on observations with the 100-m telescope at Effelsberg operated by the Max-Planck-Institut für Radioastronomie (MPIfR) on behalf of the Max-Planck-Gesellschaft.
Radio observations and magnetic studies of low-mass galaxies are difficult and thus rare to date. Single-dish observations are preferable at centimetre wavelengths as interferometric measurements of dwarfs may miss their low-level extended emission due to the missing spacings (e.g. Heesen et al. 2011). Even with the largest single radio dishes observations of lowmass galaxies are challenging. For example, Chyży et al. (2011) performed a systematic survey of dwarf galaxies in the Local Group. However, only 3 out of 12 dwarfs were radio-detected in sensitive observations with the Effelsberg $100 \mathrm{~m}$ telescope at $2.64 \mathrm{GHz}$. The results obtained indicated that the magnetic fields within the Local Group dwarfs are rather weak with a mean value of the total field strength of only $4.2 \mu \mathrm{G}$. A stronger magnetic field was only observed in the starbursting dwarf IC 10. The radio undetected dwarfs were of the lowest total mass and global star formation rate (SFR). Based on seven radio-detected low-mass dwarfs (from the Local Group and outside it), a powerlaw relation of the magnetic field strength and the surface density 
Table 1. Basic properties of the observed galaxies.

\begin{tabular}{lccccccr}
\hline \hline Name & Type $^{a}$ & $\begin{array}{c}\text { Inclination }^{b} \\
{\left[{ }^{\circ}\right]}\end{array}$ & $\begin{array}{c}\text { Size }^{a} \\
{\left[^{\prime}\right]}\end{array}$ & $\begin{array}{c}\text { Distance }^{c} \\
\text { Mpc }\end{array}$ & $\begin{array}{c}v_{\text {rot }^{b}} \\
\mathrm{~km} \mathrm{~s}^{-1}\end{array}$ & $\begin{array}{c}M_{\mathrm{HI}^{d}}{ }^{8} \\
10^{8} M_{\odot}\end{array}$ & $\begin{array}{r}M_{\text {tot }} \\
10^{9} M_{\odot}\end{array}$ \\
\hline NGC 2976 & SAc pec & 60.5 & $5.9 \times 2.7$ & 3.6 & $58.5 \pm 2.4$ & $1.5^{g}$ & 2.4 \\
NGC 3239 & IB(s)m pec & 46.8 & $5.0 \times 3.3$ & 8.3 & $95.0 \pm 2.5$ & 13.0 & 12.4 \\
NGC 4027 & SB(s)dm & 42.3 & $3.2 \times 2.4$ & $15.2^{e}$ & $97.8 \pm 4.8$ & $40.0^{h}$ & 15.5 \\
NGC 4605 & SB(s)c pec & 70.0 & $5.8 \times 2.2$ & 5.5 & $60.9 \pm 2.0$ & 2.0 & 3.9 \\
NGC 4618 & SB(rs)m & 57.6 & $4.2 \times 3.4$ & 7.8 & $65.7 \pm 4.1$ & 11.0 & 4.7 \\
NGC 5204 & SA(s)m & 58.8 & $5.0 \times 3.0$ & 4.7 & $55.9 \pm 1.1$ & 6.3 & 2.4 \\
UGC 11861 & SAB(s)dm & 75.0 & $3.5 \times 2.6$ & $20.9^{f}$ & $114.6 \pm 3.1$ & 87.0 & 31.8 \\
\hline
\end{tabular}

Notes. Data taken from ${ }^{(a)}$ NED, ${ }^{(b)}$ LEDA, ${ }^{(c)}$ Kennicutt et al. (2008), ${ }^{(d)}$ Tully et al. (1988), ${ }^{(e)}$ Bell et al. (2003), ${ }^{(f)}$ James et al. (2004), ${ }^{(g)}$ Stil \& Israel (2002), ${ }^{(h)}$ Bottinelli et al. (1982).

Table 2. Parameters of observations, integrated radio data, and thermal fraction of studied galaxies at $4.85 \mathrm{GHz}$.

\begin{tabular}{lcccccc}
\hline \hline Galaxy & $\begin{array}{c}\text { Map No. } \\
\text { 4.85 GHz }\end{array}$ & $\begin{array}{c}\sigma_{4.85}^{\mathrm{TP}} \\
\mathrm{mJy} / \mathrm{b} . \mathrm{a} .\end{array}$ & $\begin{array}{c}\sigma_{4.85}^{\mathrm{PI}} \\
\mathrm{mJy} / \mathrm{b} . \mathrm{a} .\end{array}$ & $\begin{array}{c}\mathrm{TP}_{4.85} \\
\mathrm{mJy}\end{array}$ & $\begin{array}{c}\mathrm{PI}_{4.85} \\
\mathrm{mJy}\end{array}$ & $f_{\mathrm{th}, 4.85}$ \\
\hline NGC 2976 & 14 & 0.6 & 0.1 & $32.0 \pm 2.5$ & $1.39 \pm 0.30$ & $0.31 \pm 0.07$ \\
NGC 3239 & 12 & 0.8 & 0.1 & $16.5 \pm 0.9$ & $0.22 \pm 0.14$ & $0.34 \pm 0.07$ \\
NGC 4027 & 11 & 1.5 & 0.2 & $40.7 \pm 3.2$ & $0.70 \pm 0.39$ & $0.29 \pm 0.06$ \\
NGC 4605 & 6 & 0.5 & 0.7 & $37.6 \pm 2.5$ & $<1.40$ & $0.23 \pm 0.05$ \\
NGC 4618 & 17 & 0.5 & 0.1 & $15.0 \pm 1.2$ & $0.49 \pm 0.14$ & $0.29 \pm 0.06$ \\
NGC 5204 & - & - & - & - & - & - \\
UGC 11861 & 8 & 1.5 & 0.1 & $15.1 \pm 1.5$ & $0.53 \pm 0.17$ & $0.11 \pm 0.02$ \\
\hline
\end{tabular}

of star formation rate $(\Sigma \mathrm{SFR})$ with an index of $0.30 \pm 0.04$ was proposed.

To extend that study and investigate the relations observed for dwarf galaxies, we report results from new radio polarimetric observations of seven low-mass galaxies of Magellanictype or peculiar morphology in this paper. The sizes and masses of these objects are between dwarf and typical spiral galaxies. Consequently, such galaxies have a better chance to be radiodetected than dwarfs of the Local Group and hence are suitable for further investigation of the relations found in dwarf objects (Chyży et al. 2011). In the extended sample of low-mass galaxies based on our new observations and on the published ones, we analyse the properties of total radio and polarised emissions, construct a radio-infrared correlation diagram, and investigate a generation of magnetic fields in response to the observed star formation activity. We investigate the net production of magnetic fields per single supernova explosion and relation of magnetic energy to global properties of galaxies.

\section{Sample selection and data reduction}

For the radio polarimetric observations, we selected five Magellanic-type galaxies showing large apparent optical discs $\left(>3^{\prime}\right)$ of various morphology. All of them have H I masses larger than the Large Magellanic Cloud (LMC). To provide a better coverage of galactic masses as well as a closer correspondence with the dwarf galaxies, we supplemented the sample with two peculiar galaxies (NGC 2976 and NGC 4605) with H I masses that are smaller than the LMC. The main properties of all the seven objects are summarised in Table 1. For the sake of comparison, we present not only the H I mass of galaxies but also their "indicative" total masses estimated according to the formulae: $M_{\text {tot }}=0.5 G^{-1} A v_{\text {rot }}^{2}$, where $A$ is the linear extent of the galactic major axis based on optical observations, $v_{\text {rot }}$ is the maximum velocity rotation corrected for inclination, and $G$ is the gravitational constant (cf. Karachentsev et al. 2004). Values of $A$ (in arcmin) and $v_{\text {rot }}$ are also given in Table 1 . We note that the 3D mass distribution, including the dark matter is not known for these objects and the "indicative" mass is the first order approximation of the total dynamical mass of these galaxies by assuming a spherical mass distribution.

Some galaxies in the sample have properties similar to those of spirals, for example, having an HI mass above $10^{9} M_{\odot}$ (NGC 4027) or rotational velocity larger than $100 \mathrm{~km} \mathrm{~s}^{-1}$ (e.g. UGC 11861), while others have rotational velocities and sizes similar to those of dwarf irregulars (as e.g. NGC 5404 and NGC 2976). The total "indicative" mass is largest for UGC $11861\left(3.18 \times 10^{10} M_{\odot}\right)$, which is still one order of magnitude smaller than the mass of the Andromeda galaxy $\left(3.20 \times 10^{11} M_{\odot}\right)$, when estimated in the same way. Our objects are at different stages of gravitational interaction: from galaxies that do not have distinct morphological distortions, like UGC 11861, and weakly interacting (NGC 4618) to disturbed ones (NGC 3239, see Sect. 3.1 for details).

Our radio polarimetric observations were performed with the Effelsberg $100 \mathrm{~m}$ telescope. All seven galaxies were observed at $4.85 \mathrm{GHz}$ with the dual-horn system (with the horn separation of $8^{\prime}$ ), and a correlation polarimeter in the backend by offering an output of Stokes parameters $I, Q$, and $U$. The individual coverages were obtained in both azimuth and elevation directions. To establish a flux density scale, we observed the calibration source 3C 286 and assumed its total flux as 7.45 Jy, according to the flux scale of Baars et al. (1977).

The data were reduced in NOD2 package, which combined the coverages using software beam-switching (Emerson et al. 1979) and spatial-frequency weighting methods (Emerson \& Gräve 1988). The maps were then digitally filtered to remove the spatial frequencies that correspond to noisy structures that are smaller than the telescope beam of $2.5^{\prime}$. The details of the observations are given in Tables 2 and 3. In the case of NGC 5204, the processing of the data did not bring satisfactory results due to strong artefacts of weather origin and a number of background 
W. Jurusik et al:: Magnetic fields and star formation in low-mass galaxies

Table 3. Parameters of observations, integrated radio data, and thermal fraction of studied galaxies at $8.35 \mathrm{GHz}$.

\begin{tabular}{lcccccc}
\hline \hline Galaxy & $\begin{array}{c}\text { Map No. } \\
8.35 \text { GHz }\end{array}$ & $\begin{array}{c}\sigma_{8.35}^{\mathrm{TP}} \\
\mathrm{mJy} / \mathrm{b} . \mathrm{a} .\end{array}$ & $\begin{array}{c}\sigma_{8.35}^{\mathrm{PI}} \\
\mathrm{mJy} / \mathrm{b} . \mathrm{a} .\end{array}$ & $\begin{array}{c}\mathrm{TP}_{8.35} \\
\mathrm{mJy}\end{array}$ & $\begin{array}{c}\mathrm{PI}_{8.35} \\
\mathrm{mJy}\end{array}$ & $f_{\mathrm{th}, 8.35}$ \\
\hline NGC 2976 & - & - & - & - & - & - \\
NGC 3239 & 17 & 0.4 & 0.08 & $10.4 \pm 0.8$ & $<0.2$ & $0.50 \pm 0.11$ \\
NGC 4027 & 6 & 0.7 & 0.1 & $23.7 \pm 1.1$ & $<0.2$ & $0.47 \pm 0.10$ \\
NGC 4605 & - & - & - & - & - & - \\
NGC 4618 & 16 & 0.3 & 0.08 & $13.6 \pm 0.9$ & $<0.3$ & $0.30 \pm 0.06$ \\
NGC 5204 & 4 & 0.5 & 0.1 & $8.2 \pm 0.8$ & $<0.3$ & $0.41 \pm 0.09$ \\
UGC 11861 & - & - & - & - & - & \\
\hline
\end{tabular}

sources that made it impossible to properly fit baselines in individual coverages. Therefore, the maps of NGC 5204 at $4.85 \mathrm{GHz}$ were discarded in the analysis.

A subsample of four galaxies (NGC 3239, NGC 4027, NGC 4618, and NGC 4204) was observed at $8.35 \mathrm{GHz}$ as well. At this frequency, the single-horn receiver in the secondary focus was used. High sensitivity of the observations was guaranteed by using a frequency bandwidth of $1 \mathrm{GHz}$. The objects were scanned along the RA and Dec directions. The calibrator 3C 286 was once again applied for the flux scale calibration, assuming 5.22 Jy total flux (Baars et al. 1977). We then combined individual coverages through a procedure similar to $4.85 \mathrm{GHz}$ by obtaining the final maps in Stokes $I, Q$, and $U$ with a resolution of $1.4^{\prime}$.

The final maps from both frequencies were converted to FITS format and further analysed in the AIPS program. The maps of polarised intensity, polarisation degree and polarisation position angles were thus obtained, and the rms (root mean squared) noise levels were determined (Tables 2, 3). The polarised intensity was properly corrected for the noise distribution of $Q$ and $U$ signal.

\section{Results}

\subsection{Properties of radio emission}

The distribution of radio emission at $4.85 \mathrm{GHz}$ and $8.35 \mathrm{GHz}$ and the structure of magnetic fields for all galaxies are shown in Figs. 1 and 2, respectively. The lengths of the presented $\boldsymbol{B}$-vectors are proportional to the polarised emission, and their orientation results from the observed $\boldsymbol{E}$-vectors rotated by $90^{\circ}$. We do not apply any corrections for Faraday effects: although a typical rotation measure in spiral galaxies is up to $\pm 100 \mathrm{rad} \mathrm{m}^{-2}$ (e.g. Weżgowiec et al. 2007), the corresponding rotation of the polarisation plane is up to $23^{\circ}$ at $4.85 \mathrm{GHz}$ and $7^{\circ}$ at $8.35 \mathrm{GHz}$. In case of our sample of galaxies with a regular magnetic field strength less than $2 \mu \mathrm{G}$ (Sect. 3.3), a typical density of thermal electrons in the galactic disc of $n_{\mathrm{e}}=0.03 \mathrm{~cm}^{-3}$, and a pathlength of $500 \mathrm{pc}$, the resulting rotation measure will be even smaller (about $24 \mathrm{rad} \mathrm{m}^{-2}$ ), and hence, the $\boldsymbol{E}$-vectors are expected to rotate up to about $6^{\circ}$ at $4.85 \mathrm{GHz}$.

The general radio properties of our galaxies and their relations to morphologies as seen in other ISM components are as follows:

NGC 2976 - This peculiar late-type galaxy that belongs to the M 81 group of galaxies is the smallest object in our sample (of a diameter of $6.2 \mathrm{kpc}$ ). In optical images, there is just a "pure disc" morphology with a sharp edge and without any bulge or spiral structure. Our $4.85 \mathrm{GHz}$ data show asymmetric radio emission on both sides of the optical disc with an extension to the south-west (Fig. 1a). The polarised emission is even more asymmetric with the peak shifted off the optical disc to the south. The magnetic field $\boldsymbol{B}$-vectors can be seen outside the galactic disc as parallel to its southern edge. Since the outer parts of NGC 2976 were probably undisturbed for a long time (Bronkalla et al. 1992), this configuration of the ordered field can indicate that the magnetic field was stretched or compressed by external forces acting long ago. We will analyse higher resolution VLA data for this galaxy in a future paper (Drzazga et al., in prep.).

NGC 3239 - This galaxy is gravitationally bound with six other galaxies, forming a group (Huchra \& Geller 1982). The $\mathrm{H} \alpha$ distribution is patchy (Fig. 2a), showing a possible remnant of a disc structure and (probably tidal) tails. The present $\mathrm{H} \mathrm{II} \mathrm{lu-}$ minosity function is very similar to the LMC, including a supergiant $\mathrm{HII}$ region with the luminosity of $76 \%$ of 30 Dorarus (Krienke \& Hodge 1990). The radio emission at $4.85 \mathrm{GHz}$ corresponds well to the optical disc (Fig. 1b). Some very weak polarised emission at $3 \sigma$ level $(0.24 \mu \mathrm{Jy} / \mathrm{b}$.a. $)$ is also detected between the centre and the southern optical tail. At $8.35 \mathrm{GHz}$, the strongest radio emission is associated with the brightest $\mathrm{H}$ II regions (Fig. 2a). Only the upper limit of the emission could be estimated in polarisation (Table 3).

NGC 4027 - This is a peculiar, asymmetric late-type spiral with a small, separated companion (at $\mathrm{RA}_{2000}=11^{\mathrm{h}} 59^{\mathrm{m}} 29.4^{\mathrm{s}}$, $\left.\operatorname{Dec}_{2000}=-19^{\circ} 19^{\prime} 55^{\prime \prime}\right)$. The galaxy has no bulge, and the nucleus is embedded in a bar (Eskridge et al. 2002). Its peculiar morphology and optical asymmetry is due to one of its spiral arms being bright and massive (Figs. 1c). The NGC 4027 resides in a small group of galaxies however, there is no unequivocal evidence of any encounter or merger effects. With the low resolution of our maps at 4.85 and $8.35 \mathrm{GHz}$, the radio emission covers the whole galactic disc (Figs. 1c and 2b, respectively). A patch of radio emission to the west, visible at both frequencies, has its counterpart in the NVSS map at $1.4 \mathrm{GHz}$. However, its physical relation with the galaxy is not clear. Polarised emission at $3.5 \sigma$ level is detected at $4.85 \mathrm{GHz}$ (Table 2 ) but only an upper limit of polarisation is obtained at $8.35 \mathrm{GHz}$ (Table 3 ).

NGC 4605 - The galaxy is located at the edge of M 81/M 82 group, and its rigid-body rotation creates problems in the CDM modelling of this object (Sofue 1998). Its peculiar morphology is similar to NGC 2976, which does not feature either a central component or a distinct spiral pattern. The radio emission from the galactic disc is quite noticeable at $4.85 \mathrm{GHz}$ (Fig. 1d), especially in the map with the $20 \mu \mathrm{Jy}$ background source to the south subtracted (Fig. 3a). However, the resolution of our observations did not allow us to make a more detailed comparison with the optical data. No polarised emission has been detected here.

NGC 4618 - This galaxy manifests evidence of tidal interactions: there is one prominent spiral arm to the south and a bar extending further to the southwest (Fig. 1e). The peak of total radio emission at both 4.85 and $8.35 \mathrm{GHz}$ (Fig. 2c) is set off from the brightest central region in the $\mathrm{H} \alpha$ map, towards the southern 

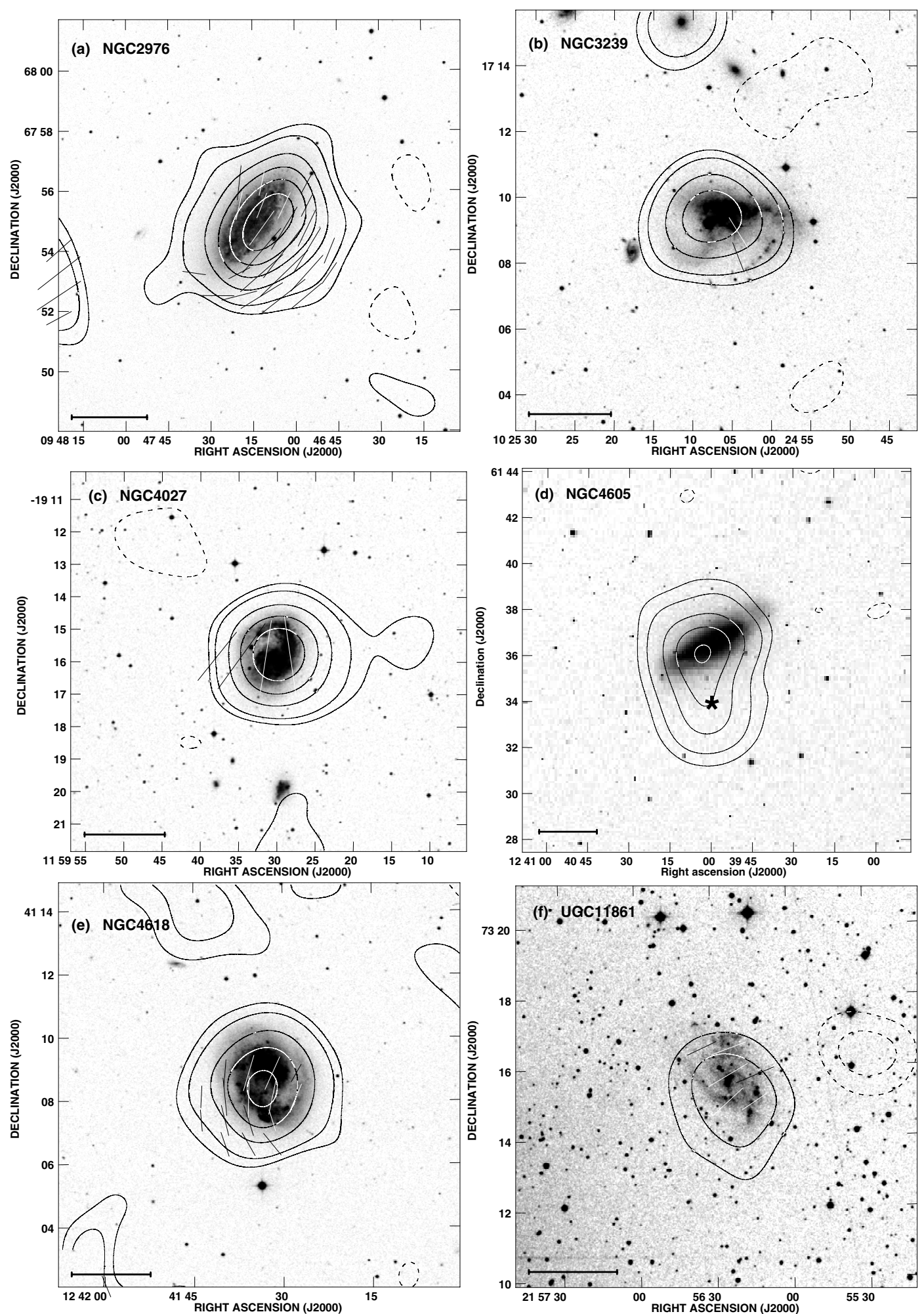

Fig. 1. Total-power contours at $4.85 \mathrm{GHz}$ and $\boldsymbol{B}$-vectors of polarised intensity of NGC $2976 \mathbf{a}$ ), NGC 3239 b), NGC $4027 \mathbf{c}$ ), NGC $4605 \mathbf{d}$ ), NGC 4618 e), UGC 11861 f) superimposed on the DSS blue images. The contour levels are as follows: 0.6 a), 0.8 b), 1.5 c), 0.5 e), 1.5 f) $\times$ $(-5,-3,3,5,10,15,20,25) \mathrm{mJy} /$ beam and $0.45 \times(-3,3,10,30,50,70) \mathrm{mJy} /$ beam d). The map resolution is $2.5^{\prime}$ HPBW, as shown as a horizontal bar at bottom left. For all the images, a vector of $1^{\prime}$ length corresponds to a polarised intensity of $0.3 \mathrm{mJy} / \mathrm{beam}$. The position of a background source subtracted from emission of NGC 4605 d) is marked by an asterisk (Sect. 3).

spiral arm. The H I distribution (Kaczmarek \& Wilcots 2012) is therefore compatible with radio emission extending from the bar to the south, which further joins a bright $\mathrm{H}$ I ring encircling the entire galaxy. The polarised radio emission at $4.85 \mathrm{GHz}$ is detected in the SE part, where the $\boldsymbol{B}$-vectors are oriented roughly along the southern optical spiral arm and resemble other tidally perturbed galaxies (Drzazga et al. 2011). The degree of polarisation of about $3 \%$ at $4.85 \mathrm{GHz}$ proves the presence of ordered magnetic fields in the outer part of the galaxy. At $8.35 \mathrm{GHz}$, a spot of polarised signal at about $3 \mathrm{rms}$ noise level is visible 

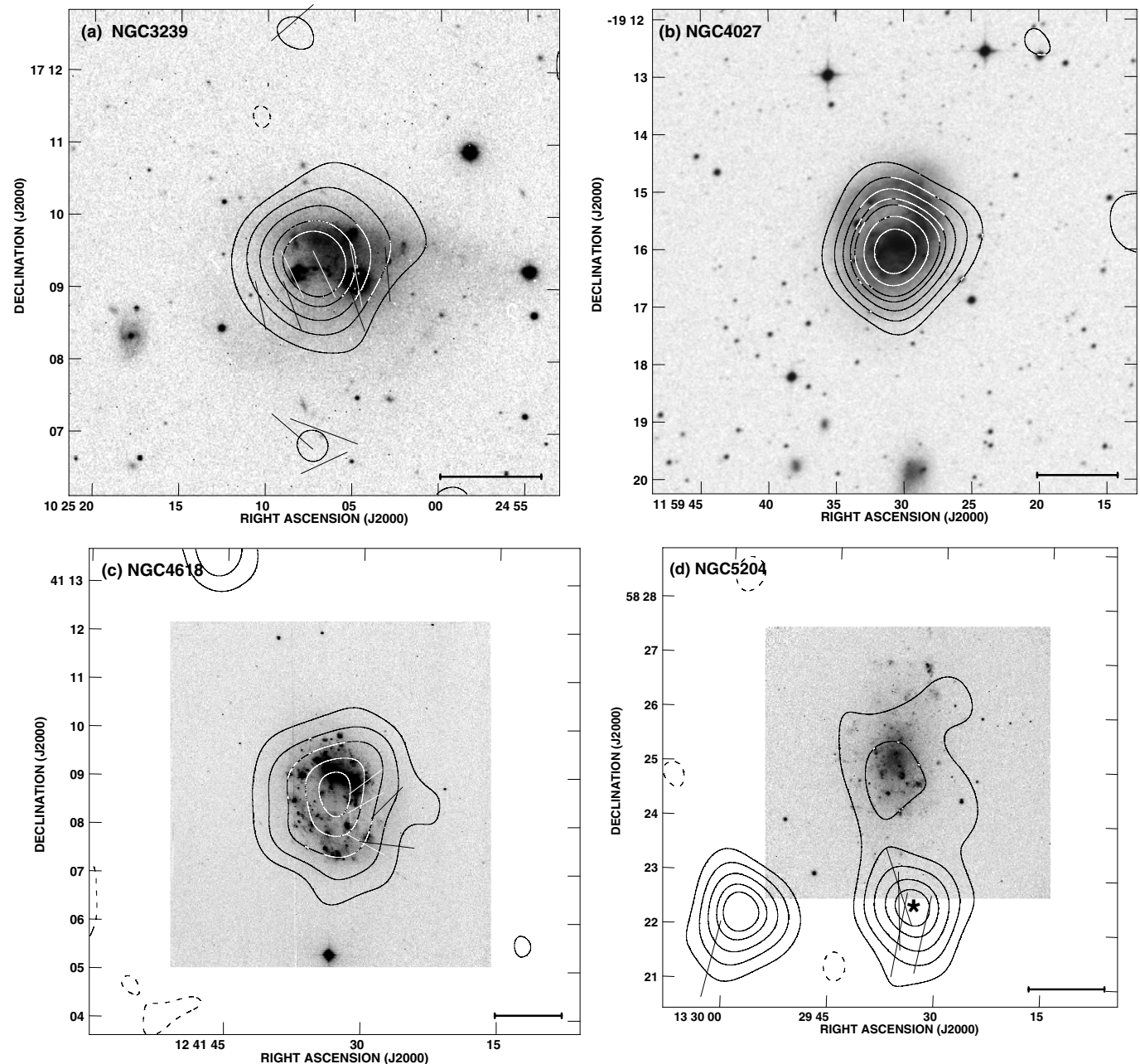

Fig. 2. Total-power contours at $8.35 \mathrm{GHz}$ and $\boldsymbol{B}$-vectors of polarised intensity of NGC 3239 a), NGC 4618 c), NGC 5204 d), superimposed on the $\mathrm{H} \alpha$ images and NGC 4027 b) superimposed on the DSS blue image. The contour levels are as follows: 0.5 a), 0.7 b), 0.3 c), 0.45 d), $\times(-5,-3$, $3,6,9,12,14,18,22) \mathrm{mJy} /$ beam. The map resolution is $1.4^{\prime} \mathrm{HPBW}$, as shown as a horizontal bar at bottom right. For all the images, a vector of $1^{\prime}$ length corresponds to the polarised intensity of $0.3 \mathrm{mJy} / \mathrm{beam}$. The $\mathrm{H} \alpha$ images of NGC 3239 and NGC 5204 are from NED and those for NGC 4618 from Knapen et al. (2004).

in the southwest part of the disc but not in the southeast part (Fig. 2c). It is possible that the noise distribution at $8.35 \mathrm{GHz}$ is not uniform in this field: this frequency is more sensitive to weather conditions and scanning effects than at $4.85 \mathrm{GHz}$. Thus, we do not consider this spot as a proper detection of polarisation at $8.35 \mathrm{GHz}$.

NGC 5204 - The galaxy is located on the periphery of M 101 group but has no close companion. The optical emission is symmetric, and the radio emission at $8.35 \mathrm{GHz}$ corresponds to it (Figs. 2d, 3b). The UV emission from the galaxy is dominated by very massive stars $\left(>30 M_{\odot}\right)$, which seems to be reflected in our data by a high thermal fraction at $8.35 \mathrm{GHz} f_{\mathrm{th}, 8.35}=0.41$ (see Sect. 3.3 and Table 3). A remarkable flattening of the radio spectrum due to significant thermal component at frequencies above $10 \mathrm{GHz}$ was claimed by Fabbiano \& Panagia (1982).

UGC 11861 - This isolated galaxy is the largest (with a diameter of $18.2 \mathrm{kpc})$ and the most massive $\left(M_{\mathrm{HI}} \approx 9 \times 10^{9} M_{\odot}\right)$ object in our sample. It shows a weak bar and a few dispersed spiral arms in optical images (Fig. 1f). The radio emission does not manifest any special features when observed with the resolution of $2.5^{\prime}$ and corresponds roughly to the optical extent of the object.
In summary, our observations indicate a substantial correspondence between radio emission and optical discs for all galaxies, regardless if the galaxy is isolated or has signs of gravitational interactions (NGC 3239, NGC 4027). Some visible shifts between maxima in radio and optical distributions (e.g. NGC 4618, Fig. 2c) may arise from more extended radio emission towards star-forming regions out of the main body of galaxy discs and from lower resolution of the radio maps. Polarised emission was detected for five out of six galaxies observed at $4.85 \mathrm{GHz}$. At $8.35 \mathrm{GHz}$, only upper $(2 \sigma)$ limits for the observed 4 galaxies were possible to be estimated (Table 3 ). The percentage of polarisation is low between $1 \%$ and $4 \%$ at $4.85 \mathrm{GHz}$. This is quite understandable when we keep in mind possible beam depolarisation due to low resolution of our single-dish observations. The low degree of polarisation may partly be intrinsic to the galaxies.

The complete determination of intrinsic configuration of magnetic fields in low-mass galaxies, as well as testing for the presence of regular (unidirectional) magnetic fields of a largescale dynamo origin, which requires high resolution information on Faraday rotation. There are no such data for our galaxies to date. They can hopefully be provided by synthesis observations (like with the JVLA) at centimetre wavelengths. 

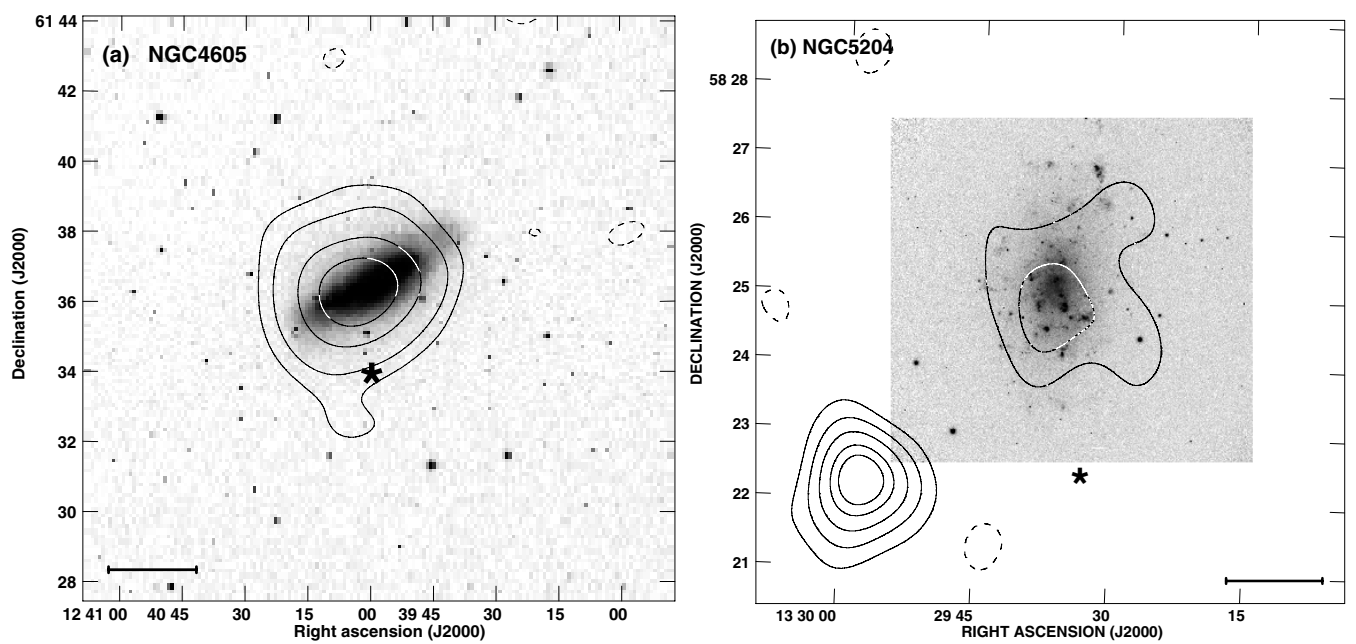

Fig. 3. Total-power contours of radio emission without nearby background sources for NGC 4605 at $4.85 \mathrm{GHz}$ a) and NGC 5204 at $8.35 \mathrm{GHz}$ b). The contour levels and other map parameters are the same as in Figs. 1 and 2. The positions of the subtracted background sources are marked by asterisks.

\subsection{Total fluxes and spectral indices}

Our observations at $4.85 \mathrm{GHz}$ or $8.35 \mathrm{GHz}$ enabled detection of all seven galaxies and measurements of their integrated fluxes in total emission and for a part of them in the polarised emission as well (Tables 2, 3). Up to now, only $1.4 \mathrm{GHz}$ observations of all these objects were available from the VLA whole sky survey (NVSS, Condon et al. 1998). We applied a special procedure to our radio maps to remove any possible influence of background sources located close to our targets on their flux measurements. Accordingly, the galaxies' neighbourhood was searched for background sources in higher resolution maps from the NVSS and FIRST surveys. In two cases, (NGC 4605 and NGC 5204) such sources were actually found. In their positions, point sources were subtracted from the maps, while their fluxes were adjusted to obtain a smooth background distribution after the subtraction (cf. Chyży et al. 2003). In the case of NGC 4605, a background source of $20 \mathrm{mJy}$ at $4.85 \mathrm{GHz}$ was subtracted at the position of RA $=12^{\mathrm{h}} 39^{\mathrm{m}} 59.4^{\mathrm{s}}$, Dec $=61^{\circ} 33^{\prime} 43^{\prime \prime}$. From the map of NGC 5204 at $8.35 \mathrm{GHz}$ a source of $8.0 \mathrm{mJy}$ was subtracted at $\mathrm{RA}=13^{\mathrm{h}} 29^{\mathrm{m}} 31.8^{\mathrm{s}}$, Dec $=58^{\circ} 22^{\prime} 24^{\prime \prime}$. The final estimated total fluxes (TP) of all galaxies are given in Tables 2 and 3. There was no need to remove background sources from the polarisation (PI) maps.

Since all our galaxies can also be found in the NVSS survey, we were able to construct simple global spectra using our data and the measured NVSS integrated fluxes. The two- or threepoint spectra obtained in this way are shown in Fig. 4. The estimated spectral index $\alpha\left(S_{v} \propto v^{-\alpha}\right)$ is in the range from 0.46 (NGC 5204) to 0.87 (NGC 4027) with an average of $0.67 \pm 0.14$. These values are close to the typical spirals for which Gioia et al. (1982) derived a mean value of $0.74 \pm 0.12$. Low-mass dwarf galaxies have typically flatter spectra and a global spectral index of around $0.38 \pm 0.20$ (Klein \& Gräve 1986). Therefore, our Magellanic-type and perturbed galaxies resemble rather massive spirals than the low-mass dwarfs and in the radio emission the synchrotron component apparently dominates over the thermal one.

\subsection{Magnetic field strengths}

The radio emission of galaxies is comprised of synchrotron and thermal (free-free) components. Having the both components

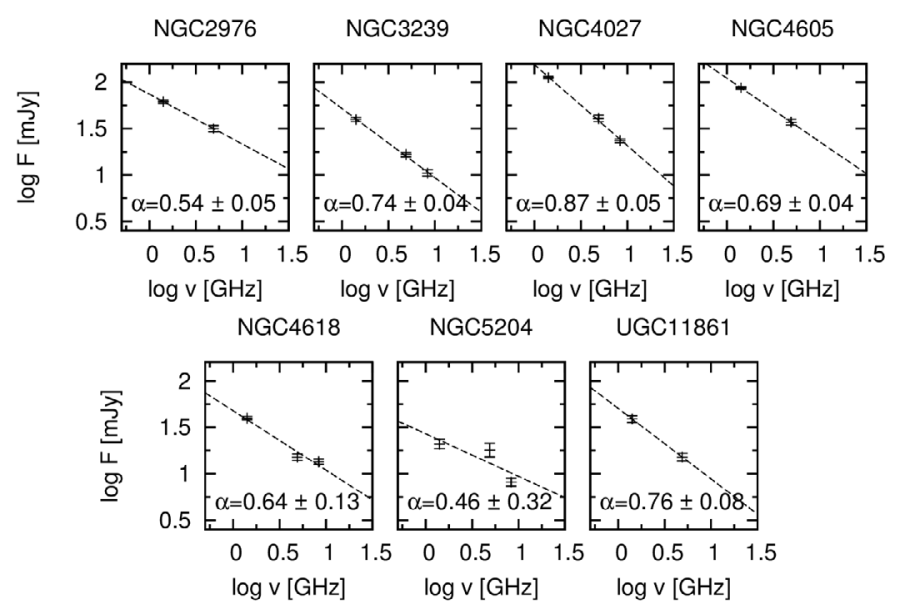

Fig. 4. Spectral indices for Magellanic-type and peculiar galaxies.

separated, the synchrotron emission can be used for estimation of strength of magnetic fields, provided energy equipartition between magnetic fields and cosmic rays is maintained (Beck \& Krause 2005). The best method to estimate the thermal component consists in modelling the nonthermal spectral index and the level of thermal fraction based on multi-frequency radio measurements. However, we cannot apply this method for our galaxies as there are not sufficient (literature) data concerning their radio measurements.

In our previous investigations of dwarf galaxies within the Local Group (Chyży et al. 2011), we determined thermal fractions from $\mathrm{H} \alpha$ fluxes, assuming that dust attenuation is negligible. While justified for dwarf galaxies, this assumption may not be valid for more massive, peculiar and Magellanic-type objects. To mitigate the effect of dust absorption, we apply a method here of correcting the observed $\mathrm{H} \alpha$ fluxes for dust attenuation, using information on the infrared (dust) emission. Such a method was proposed by Calzetti et al. (2007) to construct the most robust galactic SFR indicators, based on the observed $\mathrm{H} \alpha$ and $24 \mu \mathrm{m}$ luminosities. Having such calibration factors of the SFR (Kennicutt \& Evans 2012; Calzetti et al. 2007) and unobscured $\mathrm{H} \alpha$ fluxes at hand, we combined them with the theory of thermal emission (Caplan \& Deharveng 1986; Niklas et al. 1997). This 
Table 4. Magnetic field strength in $\mu \mathrm{G}$ for low-mass galaxies.

\begin{tabular}{lcccccc}
\hline \hline Galaxy & $\begin{array}{c}B_{\text {tot }} \\
4.85 \mathrm{GHz}\end{array}$ & $\begin{array}{c}B_{\text {ran }} \\
4.85 \mathrm{GHz}\end{array}$ & $\begin{array}{c}B_{\text {ord }} \\
4.85 \mathrm{GHz}\end{array}$ & $\begin{array}{c}B_{\text {tot }} \\
8.35 \mathrm{GHz}\end{array}$ & $\begin{array}{c}B_{\text {ran }} \\
8.35 \mathrm{GHz}\end{array}$ & $\begin{array}{c}B_{\text {ord }} \\
8.35 \mathrm{GHz}\end{array}$ \\
\hline NGC 2976 & $5.7 \pm 0.8$ & $5.5 \pm 0.8$ & $1.5 \pm 0.2$ & - & - & - \\
NGC 3239 & $6.9 \pm 0.9$ & $6.8 \pm 0.9$ & $0.9 \pm 0.1$ & $7.3 \pm 0.9$ & $7.1 \pm 0.9$ & $<2.1$ \\
NGC 4027 & $9.0 \pm 1.3$ & $8.9 \pm 1.2$ & $1.3 \pm 0.2$ & $8.1 \pm 1.2$ & $7.9 \pm 1.2$ & $<1.9$ \\
NGC 4605 & $6.4 \pm 0.9$ & $6.2 \pm 0.8$ & $<1.6$ & - & - & - \\
NGC 4618 & $6.0 \pm 0.9$ & $5.9 \pm 0.8$ & $1.3 \pm 0.2$ & $6.8 \pm 0.9$ & $6.6 \pm 0.9$ & $<1.6$ \\
NGC 5204 & - & - & - & $6.3 \pm 0.9$ & $5.9 \pm 0.8$ & $<2.2$ \\
UGC 11861 & $5.4 \pm 0.8$ & $5.3 \pm 0.8$ & $1.2 \pm 0.2$ & - & - & - \\
\hline
\end{tabular}

led us to a formula for the radio thermal emission for a galaxy at a given frequency $v$ :

$$
\begin{aligned}
F_{v}(\mathrm{mJy})= & 2.238 \times 10^{9}\left(\frac{F_{\mathrm{H} \alpha}+0.020 v_{\mathrm{IR}} F_{\mathrm{IR}}}{\operatorname{erg~s}^{-1} \mathrm{~cm}^{-2}}\right) \times\left(\frac{T_{\mathrm{e}}}{\mathrm{K}}\right)^{0.42} \\
& \times\left[\ln \left(\frac{0.04995}{v(\mathrm{GHz})}\right)+1.5 \ln \left(\frac{T_{\mathrm{e}}}{\mathrm{K}}\right)\right],
\end{aligned}
$$

where $F_{\mathrm{H} \alpha}$ is the observed $\mathrm{H} \alpha$ flux, $F_{\text {IR }}$ is the infrared flux at $24 \mu \mathrm{m}$ (or $25 \mu \mathrm{m}$ ) and $T_{\mathrm{e}}$ denotes the temperature of thermal electrons.

To calculate the radio thermal emission for our galaxies through the method described, we used the observed $\mathrm{H} \alpha$ fluxes from Das et al. (2012) for NGC 4027, James et al. (2004) for UGC 11861, and Kennicutt (2008) for the rest of galaxies. The infrared fluxes at $24 \mu \mathrm{m}$ were taken from Spitzer (Dale et al. 2009 ) or at $25 \mu \mathrm{m}$ from IRAS (Sanders et al. 2003). In our calculations we assumed that $T_{\mathrm{e}} \approx 10^{4} \mathrm{~K}$ for all the objects involved.

The obtained thermal fractions at $4.85 \mathrm{GHz}$ (see Table 2) range from 0.11 (for UGC 11861) to 0.34 (for NGC 3239). These values are similar to those for massive spiral galaxies but likely lower than for dwarfs. For the dwarfs IC 10 and NGC 6822, we derived thermal fractions at $4.86 \mathrm{GHz}$ of about $0.5-0.6$ (Chyży et al. 2003). At $8.35 \mathrm{GHz}$, the thermal fractions for our galaxies are larger (Table 3 ) and reach 0.5 for NGC 3239.

Having estimated the thermal radio emission, we separated the synchrotron component from the total radio fluxes at both frequencies. The total synchrotron emission is related to the total magnetic field, which contains two main components: the random and the ordered (uniform) one. Information on the ordered component comes from the polarised emission detected or from the upper limit of the polarised signal. Such fields can originate either from compression/shearing of turbulent random fields or from the large-scale dynamo process (Hanasz et al. 2009; Gressel et al. 2008).

On the basis of synchrotron component of the radio emission, we estimate the equipartition magnetic field strengths for all our objects (Table 4). In our calculations (cf. Beck \& Krause 2005), we assumed a constant ratio of protons to electrons $K=$ 100 , a nonthermal spectral index of 0.8 , and an unprojected synchrotron disc thickness of $L=1 \mathrm{kpc}$. We adopted the inclination angle from published data (Table 1) to calculate the effective synchrotron pathlength.

From our radio data at $4.85 \mathrm{GHz}$, the strengths of the galactic total fields averaged over galaxies are within $5.4-9.0 \mu \mathrm{G}$, while those of the ordered field are within $0.9-1.5 \mu \mathrm{G}$ (Table 4). The data at $8.35 \mathrm{GHz}$ lead to a similar conclusion, as they yield magnetic field strengths similar to those for $4.85 \mathrm{GHz}$ within the estimated uncertainties (Table 4). Hence, the values of total and ordered field strengths are both smaller than in typical spiral galaxies, where their values are about $10 \mu \mathrm{G}$ and $5 \mu \mathrm{G}$, respectively (Beck \& Wielebinski 2013). They are both larger than in typical dwarfs of the Local Group, for which the mean total field strength is $<4.2 \mu \mathrm{G}$, and the ordered field strength is $<0.9 \mu \mathrm{G}$ (Chyży et al. 2011). Therefore, by considering magnetic properties, Magellanic-type galaxies constitute an intermediate class of objects between dwarfs and massive spirals.

The observed magnetic fields were strongest for NGC 4027 and NGC 3239, which are weakly interacting (Sect. 3.1), and weakest for UGC 11861, which is an isolated galaxy. The difference (about $3 \mu \mathrm{G}$ ) is small, indicating that the influence of gravitational interaction on the magnetic field strength is rather feeble for these objects. This agrees with the results of Drzazga et al. (2011), showing that magnetic fields are only strongly enhanced for major merger systems, especially for those undergoing the phase of coalescing cores.

\subsection{Magnetic fields and SFRs}

For the purpose of a more detailed and statistically valid study of magnetic properties of low-mass objects, we enlarged our sample by including a number of cases known from the literature. Thus, we increased the number of Magellanic-type galaxies by five: three from our previous studies (NGC 4236, NGC 4656, and IC 2574; Chyży et al. 2007) and two Magellanic Clouds (LMC: Gaensler et al. 2005; SMC: Mao et al. 2008). As we aim to verify the relations found for small dwarf galaxies from our Local Group (Chyży et al. 2011), we include those LG dwarfs for which radio emission had been detected (IC 1613, NGC 6822 and IC 10) in the analysis. We add two starburst galaxies with well-defined radio properties: NGC 1569 (Kepley et al. 2010) and NGC 4449 (Chyży et al. 2000). In effect, the enlarged sample includes ten Magellanic-type galaxies, two peculiar galaxies and five dwarf irregulars, thus 17 objects in total. For a rough comparison with spiral galaxies we also analyse four massive spirals $\left(M_{\mathrm{HI}}>4 \times 10^{9} M_{\odot}\right)$ : NGC 5055, NGC 5236, NGC 6946 from the compilation of Basu \& Roy (2013) and NGC 4254 from our previous work with Chyży et al. (2007). These spirals represent the Hubble morphological types c and d.

We constructed a diagram showing the relation of total magnetic field strengths $B$ and (surface) densities of the SFR ( $\Sigma$ SFR) for all galaxies (Fig. 5). The SFRs for seven galaxies from our main sample were estimated from the SFR indicator of Calzetti et al. (2007), as mentioned in the previous section. For other galaxies from the extended sample, the SFRs were taken from the papers mentioned above. The global SFRs were then divided by the observed optical areas of galaxies (Table 1) and rescaled to give galaxy-mean $\Sigma$ SFRs in $M_{\odot} \mathrm{yr}^{-1} \mathrm{kpc}^{-2}$. The observed Magellanic-type galaxies have total magnetic field strengths and $\Sigma$ SFR between the radio-weakest dwarf galaxy IC 1613 and the starburst dwarfs NGC 1569 and IC 10 (Fig. 5). Magnetic fields in spirals are as strong as in starburst dwarfs and relatively stronger than in Magellanic-type galaxies. For the extended sample of 


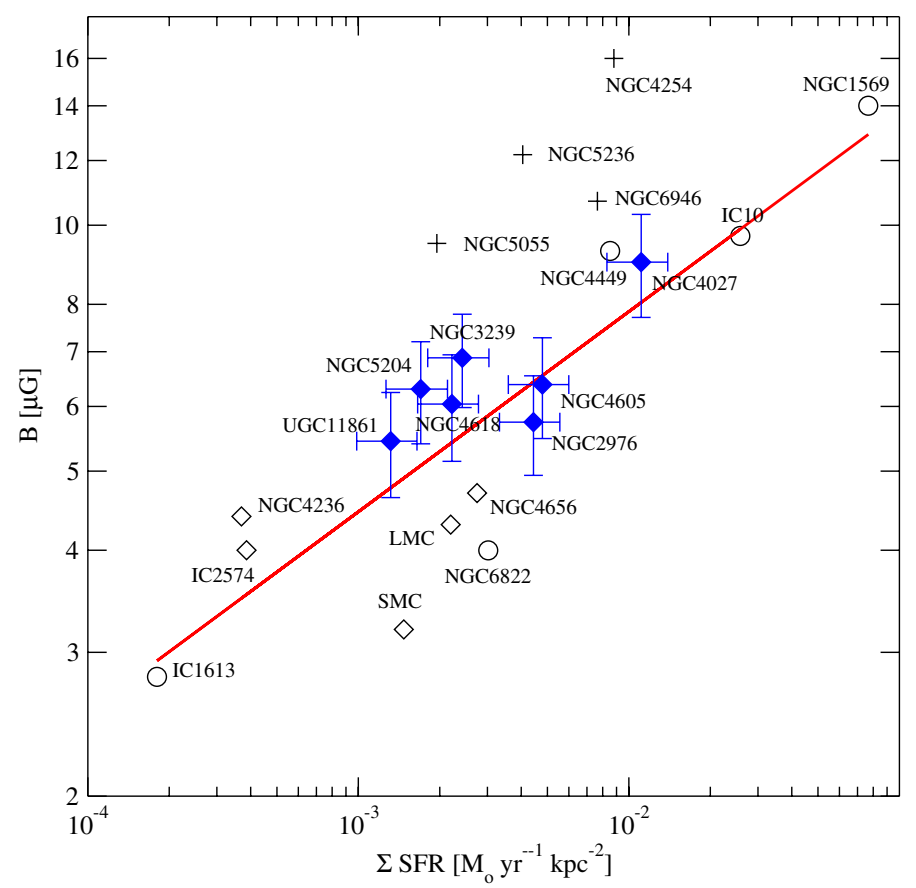

Fig. 5. Total field strength against surface density of the star formation rate $\Sigma$ SFR for the sample of galaxies of various types. Dwarfs - circles, Magellanic and peculiar low-mass galaxies - diamonds, solid diamonds - objects presented in this paper, and crosses - spiral galaxies. The solid line presents the power-law fit to 17 dwarfs and Magellanictype objects.

17 objects, the strength of the total field $B$ is strongly related with $\Sigma$ SFR, manifesting a power-law relation with an exponent $a=0.25 \pm 0.02$. This relation is statistically highly significant with a correlation coefficient $r=0.87$, and the significance level ( $p$-value of null hyphothesis) $P=5 \times 10^{-6}$. It confirms $B-\Sigma$ SFR relation found for a small sample of Local Group dwarfs with the power-law exponent of $a=0.30 \pm 0.04$ (Chyży et al. 2011). In the present analysis the statistical sample is more than twice as large than the previous one and not only supports but also even strengthens the results obtained from the Local Group dwarfs.

A similar phenomenon of magnetic field production increasing with the star-forming activity in galaxies was also found locally within individual objects - NGC 4254 with the exponent $a=0.18 \pm 0.01$ (Chyży et al. 2008) and NGC 6946 with $a=0.14 \pm 0.01$ (Tabatabaei et al. 2013). The differences in these slopes could have arisen from different contents of the ordered field and/or galaxy environment (Tabatabaei et al. 2013; Krause 2009).

We note a substantial scatter of points of about 0.5 dex to be seen in the $B-\Sigma$ SFR diagram, which could obscure such a relation if the range of values of $\Sigma$ SFR was smaller. In the present study, the pattern is distinct only when almost three orders of magnitude in $\Sigma$ SFR (Fig. 5) are considered. A significant spread in the global properties of galaxies is therefore necessary to disclose $B-\Sigma$ SFR relation.

According to our analysis, we also note that global SFR and the galactic H I mass $\left(M_{\mathrm{HI}}\right)$ are correlated $\left(r=0.74, P=10^{-4}\right)$. The estimated power-law slope for this relation $a=0.82 \pm 0.20$ from the classical regression fit (Fig. 6) and $a=1.07 \pm 0.16$ from the bisector fit. The H I mass is strongly correlated with the total galactic mass $\left(M_{\mathrm{tot}}\right.$, see Sect. 2$), r=0.92$, for our sample. Hence, a similar relation holds between the SFR and the total mass with $a=0.80 \pm 0.26$. A similar dependency with

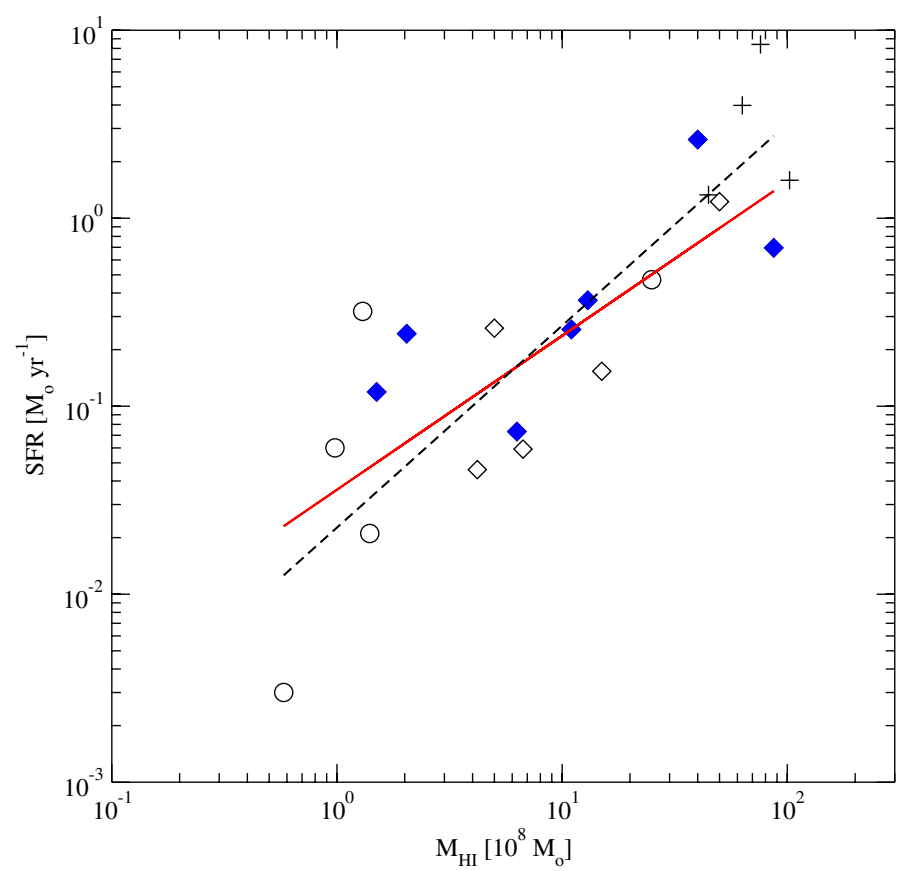

Fig. 6. Correlation of global star formation rate with $\mathrm{H}$ I mass for dwarf galaxies (circles), Magellanic-type and peculiar galaxies (diamonds) and normal spirals (crosses). Solid diamonds mark objects presented in this paper. Solid and dashed lines demonstrate the classical $Y-X$ regression fit and the bisector fit to 17 Magellanic-type and dwarf galaxies.

a slope of $\approx 1.0$ for dense molecular gas of spiral galaxies was found by Gao \& Salomon (2004). Slightly steeper $(a=1.5)$ relations were estimated for the H I mas of galaxies of various morphological types in the Local Volume (Karachentsev \& Kaisina 2013) and for dwarf galaxies in the Local Group ( $a=1.4$, Chyży et al. 2011). There are some observational and theoretical hints, in which linearly large low surface brightness galaxies can show lower SFR than the high surface brightness galaxies for the same reservoir of neutral hydrogen (Boissier et al. 2008). Consequently, differences in the star formation efficiency could cause differences in SFR-mass relations among different samples and also enlarge the spread in the observed $B-\Sigma$ SFR relation in our sample.

\section{Discussion}

\subsection{Radio-FIR correlation}

The observed connection of the magnetic field strength and the star-forming activity in galaxies can be investigated through a relation of radio and far-infrared (FIR) emission. The rates of local production of magnetic fields and CRs are tightly connected to supernova explosions of massive stars. The regions responsible for vivid star formation produce UV-photons that, in turn, cause dust heating and re-emission in the infrared band. To investigate this relation for our low-mass galaxies, we constructed a mean surface brightness radio-FIR diagram using the total $6 \mathrm{~cm}$ and $60 \mu \mathrm{m}$ emission in the radio and infrared bands, respectively (Fig. 7a). We also included bright spirals from Gioia at al. (1982) in the diagram and interacting objects from Drzazga et al. (2011) by building up a sample of 79 objects in total. As surface brightness does not depend on galaxy distance, it is an alternative way to present the radio-FIR correlation instead of luminosity. For comparison's sake, we also present classical radio-FIR relation for luminosities (Fig. 7b). In this case, statistical dependency 

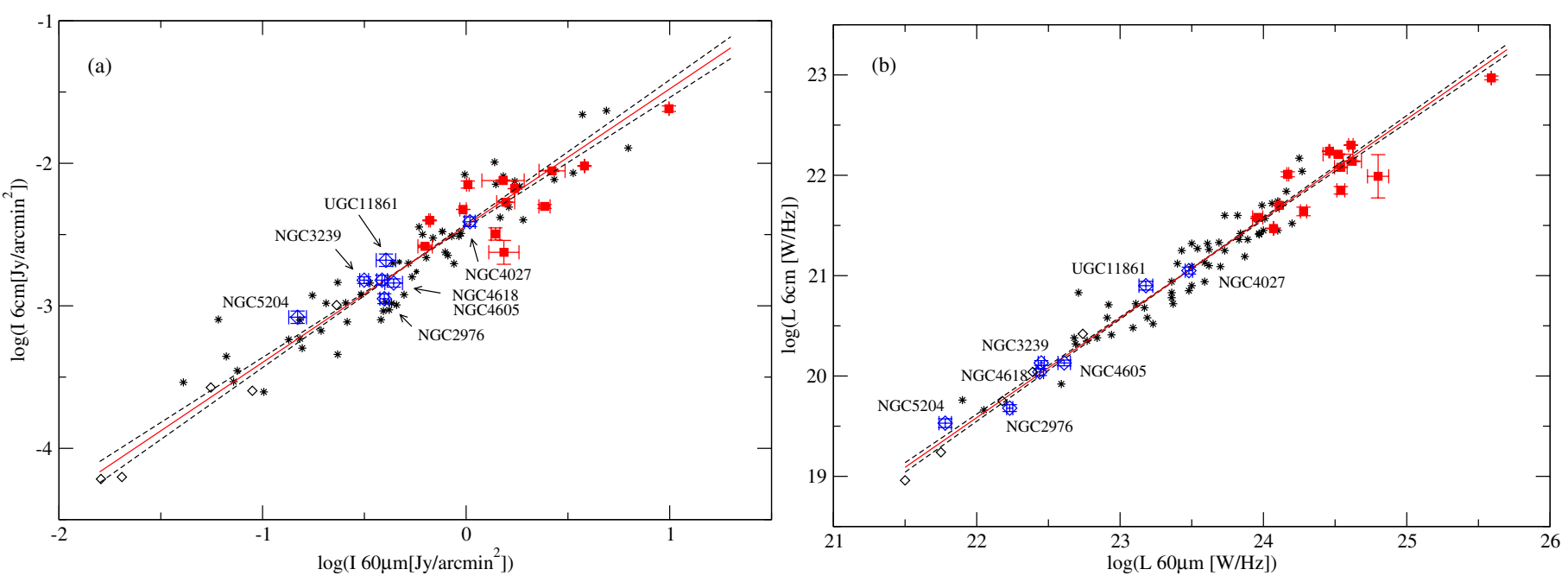

Fig. 7. Radio-FIR correlation diagram for our Magellanic-type and peculiar galaxies (diamonds), as well as interacting objects (squares) and bright spiral galaxies (asterisks). The surface brightness a) and luminosity b) at $4.85 \mathrm{GHz}(6 \mathrm{~cm})$ and at $60 \mu \mathrm{m}$ are used, respectively. The solid line is a bisector fit to all galaxies, while the dashed lines represent simple $X$ vs. $Y$ and $Y$ vs. $X$ regressions. Error bars are marked just for a few objects for example's sake.

Table 5. Power-law fits to radio - infrared relations based on surface brightness and luminosity of 79 galaxies of various types.

\begin{tabular}{lcccc}
\hline \hline Fit & \multicolumn{3}{c}{ Slopes } & Correlation \\
& $\begin{array}{c}\text { radio-FIR } \\
Y-X\end{array}$ & $\begin{array}{c}\text { FIR-radio } \\
X-Y\end{array}$ & $\begin{array}{c}\text { radio-FIR } \\
\text { bisector }\end{array}$ & \\
\hline a) Surface brightness & $0.92 \pm 0.04$ & $1.01 \pm 0.04$ & $0.96 \pm 0.03$ & 0.95 \\
b) Luminosity & $0.97 \pm 0.02$ & $1.02 \pm 0.02$ & $0.99 \pm 0.02$ & 0.98 \\
\hline
\end{tabular}

might partly arise from an obvious relation that larger galaxies are more luminous in both radio and infrared bands. The obtained slopes in the power-law fits and correlation coefficients are shown in Table 5 for both kinds of relations.

Our investigation shows that Magellanic-type and peculiar galaxies do not deviate in any systematic way from the powerlaw fit constructed for all galaxies, including interacting objects (Drzazga et al. 2011). This statement is valid for the relation constructed for surface brightness (which shows a slope of $0.96 \pm 0.03$ from the bisector fit) and for luminosity (with a slope of $0.99 \pm 0.02$ ). The radio-FIR relation for luminosity is tighter: regression lines are closer to each other and the correlation coefficient is slightly larger $(r=0.98)$ than for the surface brightness relation $(r=0.95)$, as expected (Table 5).

According to the above argumentation, the radio-FIR correlation is thought to be based on the connection between total (mostly turbulent) magnetic fields and star-formation activity, as shown in Fig. 5. Therefore, a small-scale field amplification producing random magnetic fields must work in the Magellanic-type galaxies, likewise, as in more massive and interacting objects.

We note that the slopes of the constructed relations were obtained for the total radio emission, which is a sum of thermal and nonthermal ones. Careful studies of local radio-FIR relation in individual galaxies revealed slightly steeper slopes for thermal radio emission and flatter slopes for synchrotron emission (e.g. Hoernes \& Berkhuijsen 1998; Hughes et al. 2006; Tabatabaei et al. 2013). The differences in slopes are probably caused by CRs propagation effects, properties of the magnetic field, and the existence of a thick disc/halo in galaxies (Berkhuijsen et al. 2013).

The low-emission objects are of particular interest in the constructed radio-FIR relations. For a large sample of galaxies
Yun et al. (2001) noticed a systematically diminishing radio emission (at $1.4 \mathrm{GHz}$ ) for objects of the lowest infrared luminosity. These galaxies showed a systematic tendency to lie below the best-fit line of the radio-FIR relation. Although our sample contains low-mass objects manifesting weak magnetic field strength, no distinct radio deficiency at $4.85 \mathrm{GHz}$ can be seen in the radio-FIR relations (Fig. 7). We therefore estimated radio luminosities at $1.4 \mathrm{GHz}$ for our galaxies based on the NVSS survey to check their relation to Yun et al.'s outliers. It turned out that our galaxies have luminosities starting from $5 \times 10^{19} \mathrm{~W} \mathrm{~Hz}^{-1}$ at $1.4 \mathrm{GHz}$ and hence are located just above the break in the radioFIR relation provided by Yun et al. We can still conjecture that deviations from the general radio-FIR relation are to be expected for our low-mass objects at much lower radio frequencies due to various processes shaping low-energy CRs spectra. A statistical study of such processes will soon be possible with all-sky LOFAR surveys (Röttgering et al. 2011).

We can thus conclude that the low-mass stellar systems of Magellanic or peculiar morphology in our local neighbourhood feature similar physical conditions as star formation, magnetic field, and cosmic-ray generation processes as those observed for massive or interacting galaxies.

\subsection{Production of magnetic energy}

The radio-FIR relation reveals some universal physical processes at work in the galactic ISM on small and large scales. Although this relation is not fully accounted for (e.g. Roychowdhury \& Chengalur 2012; Schleicher \& Beck 2013), one can expect that the observed (correlated) different levels of galactic radio and infrared emission result from different rates of supernova explosions. However, it is not well known yet if the production of magnetic field energy per supernova event is independent from 
various properties of galaxies, such as the global SFR, galactic mass, or morphology. The supernova energy release is an internal process and should not depend on the environment. However, the production of magnetic fields is the result of a chain of physical processes whose efficiencies may depend on local conditions in the ISM (see e.g. Gent et al. 2013). For example, the turbulence driven by supernova, which by the fluctuation dynamo produces magnetic fields, is the process intrinsically related to the ISM. The magnetic energy production is also related to the production of CRs (cf. equipartition assumption) which results from local acceleration processes in the diffusive shock of the supernova remnant. The MHD simulations also suggest that fluctuation of the SFR may influence the dynamo action and magnetic fields (Hanasz et al. 2004). The enhancement of magnetic fields on large scales also depends on galactic rotation and galactic mass. Hence, the problem of the net production of magnetic field per supernova explosion is an important issue for our understanding of generation mechanisms of magnetic fields in various types of galaxies and for building up reliable MHD models of galactic evolution.

We investigate this issue for all objects in our extended sample of dwarfs, Magellanic, peculiar, and spiral galaxies. We calculated a mean value of the energy (volume) density of magnetic fields $u_{B}$ for each studied galaxy from the estimated total magnetic field strength: $u_{B}=B^{2} /(8 \pi)$. Contrary to the synchrotron emission and the surface density of the SFR, it is worth to notice here that, the magnetic field strength is a purely local value, which is not integrated over the galactic pathlength (cf. Sect. 3.4).

The galactic supernova rate $v_{\mathrm{SN}}$ depends on the stellar initial mass function (IMF). In the case of the Salpeter IMF, it takes a form of a single power-law with an index $\gamma=2.35$. Following Kennicutt (1998) for the low- and high-mass limits of the IMF, we apply $0.01 M_{\odot}$ and $100 M_{\odot}$, respectively. Only stars more massive than $8 M_{\odot}$ finish their evolution as supernovae, depositing large amounts of energy in the ISM, which produces CRs in their shocks and enhancing magnetic fields. Under these conditions, the global rate of the supernova explosions (per year) scales for a given galaxy with the star formation rate as $v_{\mathrm{SN}}=0.02 \mathrm{SFR}$.

The observed synchrotron emission of galaxies results not only from the very recent supernova explosions described by the current SFR but also from the earlier ones, whose magnetic fields have not dissipated yet by turbulent diffusion. A crude estimation of this time can be made from a characteristic diffusion time scale $\tau=L^{2} / \eta$, where $\eta=1 / 3 v L$ is the turbulent magnetic diffusivity, $v$ is the characteristic turbulent velocity, and $L$ is the magnetic field eddy scale. Hence, the magnetic energy produced per supernova event is

$U_{B}^{\mathrm{SN}}=\frac{u_{B} V}{v_{\mathrm{SN}} \tau}=\frac{B^{2} a^{2} h}{8 v_{\mathrm{SN}} \tau}$,

where $V=\pi a^{2} h$ is the approximate galaxy disc volume with a radius $a$ and a scale height $h$. In our approximations for the galactic radius, we apply the linear size of the major axis (Table 1 ) and a value of $1 \mathrm{kpc}$ or $0.5 \mathrm{kpc}$ for the height $h$, depending on whether the observed galactic minor axis is larger or smaller than $1 \mathrm{kpc}$. We assume the turbulent velocity of the ISM $v \approx 10 \mathrm{~km} \mathrm{~s}^{-1}$ and approximate the magnetic field scale in the ISM as $L \approx 100 \mathrm{pc}$, which gives $\eta \approx 10^{26} \mathrm{~m}^{2} \mathrm{~s}^{-1}$.

The obtained diagram, which shows magnetic energy per supernova event $U_{B}^{\mathrm{SN}}$ across different levels of global SFR, is presented in Fig. 8. There is no specific relation to be seen (the

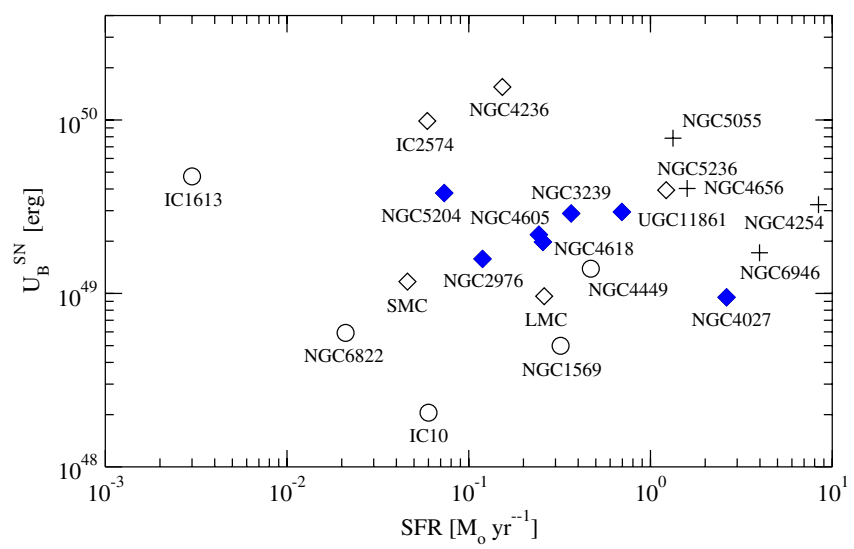

Fig. 8. Production of magnetic energy per individual supernova event $U_{B}^{\mathrm{SN}}$ for dwarf (circles), Magellanic-type and peculiar galaxies (diamonds) and normal spirals (crosses) of different SFR. The objects presented in this paper are marked with solid diamonds.

estimated slope is $-0.04 \pm 0.13)$, and the correlation coefficient is not significant either $(r=-0.07, P=0.77)$. The roughly constant magnetic field production per supernova event for a variety of objects from dwarfs and Magellanic-type objects to massive galaxies, actually suggests that the same processes are at work in the ISM in all these objects. In this scenario, starburst dwarf galaxies and large, massive galaxies can produce stronger magnetic fields just because supernova explosions are denser in these objects in time or in space. For example, the slowest formingstars object in the analysed sample, IC 1613, shows the same production of magnetic energy $\left(4 \times 10^{40} \mathrm{erg}\right)$ as NGC 4656, which has two and half orders of magnitude larger star formation rate.

Close inspection of the relation also gives some hints that among galaxies of the same SFR, like IC 2574 and IC 10, those which are larger in size (like IC 2574) produce larger magnetic energy density per single supernova event. The same is observed for several other galaxies. If it is not caused by a selection effect, this would suggest that the most effective production of magnetic fields energy density occurs in less compact star-forming regions and in larger galaxies of low-surface brightness. Indeed, the power-law fit shows weak dependence $U_{B}^{\mathrm{SN}} \propto(S F R / V)^{-0.58 \pm 0.06}$ with high negative correlation $(r=-0.9)$. This effect could be caused by a stronger diffusion of $\mathrm{CR}$ electrons or/and stellar winds from more compact star formation regions with clustered supernovae. This effect also resembles the results of MHD modelling that too strong star-forming activity in galaxies can quench magnetic fields production (Hanasz et al. 2004).

Our calculations also provide an independent estimation of the value of average magnetic energy release per supernova event: $E_{B}^{\mathrm{SN}} \approx 3 \times 10^{49} \mathrm{erg}$ (Fig. 8). If the supernova explosion exhausts the canonical total kinetic energy of about $10^{51} \mathrm{erg}$, then the production of magnetic energy is at the level of about $3 \%$ of this total energy. This is an independent estimation of the parameter that needs to be assumed in MHD simulations of magnetic field evolution in galaxies (e.g. Hanasz et al. 2009; Gressel et al. 2008).

Furthermore, we compared total magnetic field energy in galaxies using the equation $U_{B}^{\text {tot }}=u_{B} V$ and galactic H I mass (Fig. 9). In this case, a strong power-law relation (with an index of $1.09 \pm 0.16$ ) is clearly discernible and quantitatively confirmed by the large correlation coefficient $r=0.86(P=$ $\left.9 \times 10^{-6}\right)$. This relationship can be divided by the galactic volume, which yields an approximate dependence of magnetic field 
W. Jurusik et al.: Magnetic fields and star formation in low-mass galaxies

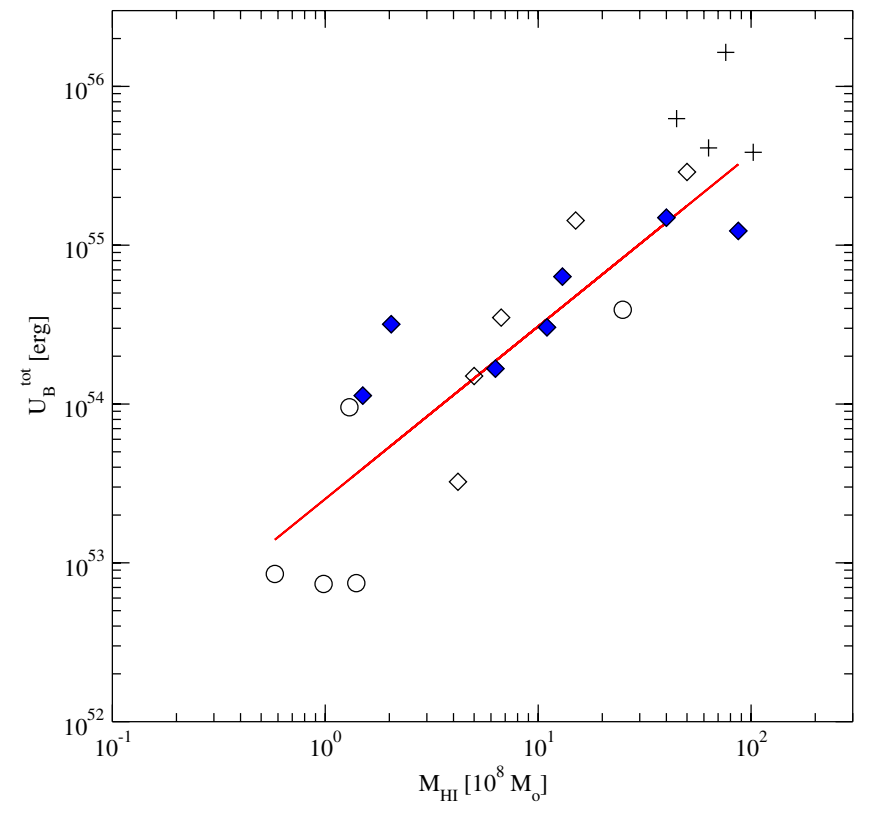

Fig. 9. Total magnetic energy $U_{B}^{\text {tot }}$ versus galactic H I mass. Circles dwarf galaxies, diamonds - Magellanic-type and peculiar galaxies, crosses - normal spirals, and solid diamonds - the objects are presented in this paper. The solid line presents the power-law fit to the extended sample of 17 dwarfs and Magellanic-type objects.

strength on the local H I gas density: $B \propto \rho_{\mathrm{HI}}^{0.54}$. We checked this relationship for the total gas masses of galaxies $M_{\text {gas }}$, which includes both the atomic and molecular gas phases, as determined by the simple relation $M_{\text {gas }}=1.3 \times\left(M_{\mathrm{HI}}+M_{\mathrm{H}_{2}}\right)$ (Boselli 2012). For 10 out of 17 galaxies, we found $\mathrm{H}_{2}$ masses in the literature. For the rest (NGC 3239, NGC 4027, NGC 4605, NGC 4618, NGC 5204, UGC 11861, and NGC 4656), we estimated the $M_{\text {gas }}$ from the gas-to-dust ratio and the dust mass from $100 \mu \mathrm{m}$ IRAS data, according to the method outlined by Boselli (2012). For this, case the power-law fit to the $U_{B}^{\text {tot }}-M_{\text {gas }}$ relation gives an exponent of $1.14 \pm 0.12\left(r=0.88, P=2 \times 10^{-7}\right)$, which results in a $B \propto \rho_{\text {gas }}^{0.57}$ relationship. The values of the exponent in the $B$-gas density relations for both the $\mathrm{HI}$ and the total gas agree within the statistical uncertainties with the theoretical value of 0.5 for equipartition of the magnetic field energy and the turbulent energy of the ISM. Such an equipartition was also found in MHD simulations of Cho \& Vishniac (2000). A slightly smaller slope of $0.48 \pm 0.05$ for the total cool gas $\left(\mathrm{HI}\right.$ plus $\left.\mathrm{H}_{2}\right)$ and the full range of galactic morphology, including high surface-brightness and interacting galaxies, was reported by Niklas \& Beck (1997).

The $B$-gas density relation represents a portion of processes leading to the observed radio-FIR correlation. In addition, the correlations involve other processes, like coupling between magnetic fields and CRs, the production of CRs (cf. equipartition assumption), the Schmidt-Kennicutt law, and dust re-radiation of UV photons, giving FIR indicators of the SFR (Basu et al. 2012). All the presented results prove that the low-mass galaxies from the analysed sample show several common patterns for whole galaxies. To better understand these processes, a future analysis of more massive galaxies is desirable to cover a wider span of analysed parameters and to allow multi-dimensional statistical analysis.

\section{Conclusions}

We performed radio polarimetric observations of seven lowmass galaxies with the Effelsberg $100 \mathrm{~m}$ telescope at $4.85 \mathrm{GHz}$ and/or 8.35 GHz. Five galaxies are classified as Magellanic-type and two (NGC 2976 and NGC 4605) represent a class of peculiar "pure disc" objects with low mass.

We found the following:

- The observations of all seven galaxies indicate that their radio emission closely follow optical properties of the discs, regardless of whether galaxies are isolated or show signs of tidal interactions.

- For the observed galaxies, radio thermal fractions range from 0.11 to 0.34 at $4.85 \mathrm{GHz}$ and correspond more to large spirals than to dwarf galaxies. Similarly, radio spectral indices with a mean of about 0.67 are closer to spiral galaxies $(\approx 0.74)$ than to dwarf objects $(\approx 0.38)$.

- The estimated strengths of the total magnetic field is in the range of $5-9 \mu \mathrm{G}$ and those of the ordered fields $1-2 \mu \mathrm{G}$. They are significantly weaker than for typical spirals. At $4.85 \mathrm{GHz}$, the polarised emission was detected for five out of six galaxies, showing a small polarisation degree of only $1-4 \%$.

- Our analysis of the extended sample of 17 galaxies shows that the Magellanic-type galaxies fit well into the pattern obtained for dwarf galaxies in the Local Group (Chyży et al. 2011), indicating that the production of turbulent magnetic field is related to the surface density of the SFR with a powerlaw index of $0.25 \pm 0.02$. Similar patterns of magnetic field production were also found to work locally within single galaxies (Tabatabaei et al. 2013; Chyży et al. 2008).

- The Magellanic-type galaxies from our extended sample hold the general radio $(4.85 \mathrm{GHz})$ and far-infrared $(60 \mu \mathrm{m})$ power-law relation, which is determined for surface brightness of galaxies of various types with a slope of $0.96 \pm 0.03$ and correlation coefficient $r=0.95$. The ascertained farinfrared relation based on luminosity of galaxies is tighter and steeper (with a slope of $0.99 \pm 0.02$ and $r=0.98$ ) but likely includes a partial correlation from a simple tendency of larger objects, which is also more luminous in both radio and infrared bands.

- The estimated production of magnetic field energy per supernova event in low-mass galaxies does not depend on either the galactic global SFR $(r=-0.07, P=0.77)$, on morphological type, or on galactic mass and is roughly constant for all the objects. We estimated magnetic energy release as about $3 \%\left(3 \times 10^{49} \mathrm{erg}\right)$ of the total available kinetic energy in an individual supernova explosion (about $10^{51} \mathrm{erg}$ ).

- The total magnetic field energy of our galaxies scales almost linearly with the galactic H I mass (with the power-law index of $1.09 \pm 0.16)$ and the total atomic and molecular gas mass (with the exponent of $1.14 \pm 0.12$ ). This results in scaling of the total magnetic field strength with the local density of $\mathrm{HI}\left(B_{\text {tot }} \propto \rho_{\mathrm{HI}}^{0.54}\right)$ and total H I $+\mathrm{H}_{2}$ gas $\left(B \propto \rho_{\text {gas }}^{0.57}\right)$. Such relations indicate equipartition of the magnetic energy and the turbulent kinetic energy of the gas. This supports the conclusion that the total magnetic field is dominated by the turbulent component, which results from the small-scale dynamo process rather than from the large-scale one.

Acknowledgements. We thank an anonymous referee for helpful comments and suggestions. This research has been supported by the scientific grant from the Polish National Science Centre $(\mathrm{NCN})$, decision no. DEC-2011/03/B/ST9/01859. R.B. and U.K. acknowledge support from DFG Research Unit FOR1254. We acknowledge the use of the HyperLeda (http:// leda.univ-lyon1.fr) and NED (http://nedwww.ipac.caltech.edu) databases. 


\section{References}

Baars, J. W. M., Genzel, R., Pauliny-Toth, I. K. K., \& Witzel, A. 1977, A\&A, 61,99

Basu, A., \& Roy, S. 2013, MNRAS, 433, 1675

Basu, A., Roy, S., \& Mitra, D. 2012, ApJ, 756, 141

Beck, R., \& Krause, M. 2005, Astron. Nachr., 326, 414

Beck, R., \& Wielebinski, R. 2013, in Planets, Stars and Stellar Systems, eds.

D. T. Oswalt, \& G. Gilmore (Dordrecht: Springer), 5, 641

Bell, E. F. 2003, ApJ, 586, 794

Berkhuijsen, E. M., Beck, R., \& Tabatabaei, F. S. 2013, MNRAS, 435, 1598

Boissier, S., de Paz, A. G., Bodelli, A., et al. 2008, ApJ, 681, 244

Boselli, A. 2012, A panchromatic view of galaxies (Wiley-VCH)

Bottinelli, L., Gouguenheim, L., \& Paturel, G. 1982, A\&A, 113, 61

Bronkalla, W., Notni, P., \& Mutter, A. A. R. 1992, Astron. Nachr., 313, 320

Calzetti, D., Kennicutt, R. C., Engelbracht, C. W., et al., 2007, ApJ, 666, 870

Caplan, J., \& Deharveng, L. 1986, A\&A, 155, 297

Cho, J., \& Vishniac, E. T. 2000, ApJ, 539, 273

Chyży, K. T. 2008, A\&A, 482, 755

Chyży, K. T., Beck, R., Kohle, S., Klein, U., \& Urbanik, M. 2000, A\&A, 355, 128

Chyży, K. T., Knapik, J., Bomans, D. J., et al. 2003, 405, 513

Chyży, K. T., Bomans, D. J., Krause, M., et al. 2007, A\&A, 462, 933

Chyży, K. T., Weżgowiec,M., Beck, R., \& Bomans, D. 2011, A\&A, 529, A94

Condon, J. J., Cotton, W. D., Greisen, E. W., et al. 1998, AJ, 115, 1693

Dale, D. A., Cohen, S. A., Johnson, L. C., et al., 2009, ApJ, 703, 517

Das, M., Sengupta, C., Ramya, S., \& Misra, K. 2012, MNRAS, 423, 3274

Drzazga, R. T., Chyży, K. T., Jurusik, W., \& Wiórkiewicz, K. 2011, A\&A, 533, A22

Emerson, D. T., \& Gräve, R. 1988, A\&A, 190, 353

Emerson, D. T., Klein, U., \& Haslam, C. G. T. 1979, A\&A, 76, 92

Eskridge, P. B., Frogel, J. A., Pogge, R. W., et al. 2002, ApJS, 143, 73

Fabbiano, G., \& Panagia, N. 1982, ApJ, 266, 568

Gaensler, B. M., Haverkorn, M., Staveley-Smith, L., et al. 2005, Science, 307, 1610

Gao, Y., \& Solomon, P. M. 2004, ApJS, 152, 63

Gent, F. A., Shukurov, A., Fletcher, A., Sarson, G. R., \& Mantrere, M. J. 2013, MNRAS, 432, 1396
Gioia, I. M., Gregorini, L., \& Klein, U. 1982, A\&A, 116, 164

Gressel, O., Elstner, D., Ziegler, U., Rüdiger, G. 2008, A\&A, 486, L35

Hanasz, M., Kowal, G., Otmianowska-Mazur, K., \& Lesch, H. 2004, ApJ, 605, L33

Hanasz, M., Otmianowska-Mazur, K., Kowal, G., \& Lesch, H. 2009, A\&A, 498, 335

Heesen, V., Rau, U., Rupen, M. P., Brinks, E., \& Hunter, D. A. 2011, ApJ, 739, L23

Hoernes, P., Berkhuijsen, E. M., \& Xu, C. 1998, A\&A, 334, 57

Huchra, J. P., \& Geller, M. J. 1982, ApJ, 257, 423

Hughes, A., Wong, T., Ekers, R., et al. 2006, MNRAS, 370, 363

James, P. A., Shane, N. S., Beckman, J. E. et al. 2004, A\&A, 414, 23

Kaczmarek, J. F., \& Wilcots, E. M. 2012, AJ, 144, 67

Karachentsev, I. D., \& Kaisina, E. 2013, AJ, 146, 46

Karachentsev, I. D., Karachentseva, V., Huchtmeier, W. K., \& Makarov, D. I. 2004, AJ, 127, 2031

Kennicutt, R. C. 1998, ApJ, 498, 541

Kennicutt, R. C., \& Evans, N. J. 2012, ARA\&A, 50, 531

Kennicutt, R. C., Lee, J. C., Funes, S. J., et al. 2008, ApJ, 178, 247

Kepley, A. A., Mühle, S., Everett, J., et al. 2010, ApJ, 712, 536

Klein, U., \& Gräve, 1986, A\&A, 161, 155

Knapen, J. H., Stedman, S., Bramich, D. M., Folkes, S. F., \& Bradley, T. R. 2004, A\&A, 426, 1135

Krause, M. 2009, in Rev. Mex. Astron. Astrofis. Conf. Ser., 36, 25

Krienke, K., \& Hodge, P. 1991, PASP, 103, 268

Mao, S. A., Gaensler, B. M., Stanimirović, S. et al. 2008, ApJ, 688, 1029

Niklas, S., \& Beck, R. 1997, A\&A, 320, 54

Roychowdhury, S., \& Chengalur, J. N. 2012, MNRAS, 423, L127

Röttgering, H., Afonso, J., Barthel, P., et al. 2011, J. Astrophys. Astron., 32, 557

Sanders, D. B., Mazzarella, J. M., Kim, D.-C, et al. 2003, AJ, 126, 1607

Schleicher, D. R. G., \& Beck, R. 2013, A\&A, 556, A142

Sofue, Y., Tomita, A., Tutui, Y., Honma, M., \& Takeda, Y. 1998, PASJ, 50, 427 Stil, J. M., \& Israel, F. P. 2002, A\&A, 389, 29

Tabatabaei, F. S., Schinnerer, E., Murphy, E. J., et al. 2013, A\&A, 552, A19

Tully, R. B. 1988, Nearby Galaxies Catalogue (Cambridge Univ. Press)

Weżgowiec, M., Urbanik, M., Vollmer, B., et al. 2007, A\&A, 471, 93

Yun, M. S., Reddy, N. A., Condon, J. J. 2001, AJ, 554, 803 